\title{
Primordial Rotation of the Universe, Hydrodynamics, Vortices and Angular Momenta of Celestial Objects
}

\author{
C Sivaram ${ }^{1}$ and Kenath Arun $^{2}{ }^{2}$ \\ ${ }^{1}$ Indian Institute of Astrophysics, Bangalore, 560 034, India \\ ${ }^{2}$ Christ Junior College, Bangalore, 560 029, India
}

\begin{abstract}
The origin of rotation or spin of objects, from stars to galaxies, is still an unanswered question. Even though there are models which try to explain this, none of them can account for the initial impulse that gave rise to this spin. In this paper we present that a cosmological model that contains a term involving the primordial spin of the universe can explain how these objects acquired the property of spin. This model also gives a natural explanation for the quadratic scaling of angular momentum with mass. Currently no cosmological model indicates as to why there are hundred billion galaxies with hundred billion stars in each. In this paper we invoke the property of non-irrotational hydrodynamic flow in order to explain how a primordial rotation of the universe broken up into vortex line structures, can indeed lead to formation of a large number of galactic structures and these in turn can lead to equally large number of stars within each galaxy. Again, from this model, the background torsion due to a universal spin density not only gives rise to angular momenta for all structures but also provides a background 'centrifugal term' acting as a repulsive gravity accelerating the universe, with spin density acting as effective cosmological constant.
\end{abstract}

Keywords: Primordial cosmic rotation, hydrodynamics, vortices, angular momenta, cosmological constant.

\section{INTRODUCTION}

One of the biggest unanswered questions in astrophysics is "what is the origin of the rotation (angular momentum) or spin of all objects: from galaxies, stars, star clusters, etc.; and are they related to basic quantum properties of elementary particles?" Currently there are elaborate models that attempt to explain the angular momentum of objects ranging from planets to clusters of galaxies; however, none explain where the initial impulse moment comes from. The same dilemma applies for the spin of all objects - stars, galaxies, etc. [1,2].

\section{PRIMORDIAL COSMIC ROTATION}

One possible explanation as to how all objects acquired the property of spin could be cosmological models which also contain a term involving the primordial spin of the universe. In homogenous and isotropic models, universe with matter may not only expand but also rotate [3-5] (relative to local gyroscope).

A Friedmann equation including a $\omega^{2}$ term has been used by several authors. Metric is modified as:

$$
d s^{2}=\left(1-\frac{\omega^{2} r^{2}}{c^{2}}\right) c^{2} d t^{2}-R^{2}(t)\left[\frac{d r^{2}}{1-k r^{2}}+r^{2} d \Omega^{2}\right] ; \quad d t . d \phi
$$

terms are neglected (as they are off-diagonal terms).

The spin is introduced through the torsion tensor. The Lagrangian has the same form as the Hilbert one of GR, but

*Address correspondence to this author at the Christ Junior College, Bangalore, 560 029, India; Tel: +91-80-4012 9292; Fax: +91-80- 4012 9222;

E-mail: kenath.arun@cjc.christcollege.edu with curvature scalar $\mathrm{R}$ also containing the torsion scalar $Q=\frac{4 \pi G \sigma}{c^{2}}, \sigma$ the spin density, i.e. we have: $\frac{1}{16 \pi G} \int R \sqrt{-g}, \mathrm{R}$ being constructed from anti-symmetric connections. The R$\mathrm{W}$ metric can still be used.

The torsion can also be related to the gradient of a scalar $Q \rightarrow \partial_{\mu} \phi$, in which case the action is equivalent to a scalartensor theory $[6,7,8]$.

A general solution including the rotation is given by:

$\frac{\dot{R}^{2}}{R^{2}}-\frac{8 \pi G \rho}{3}+\omega^{2}=0$

For the last two terms to be comparable:

$\frac{8 \pi G \rho}{3}=\omega^{2}$

This only fixes the upper value of $\omega$. The idea is that at earlier epochs, the matter term was dominant, but when density drops the $\omega$ term becomes comparable. The universe dynamics changed from deceleration to acceleration, thus giving an alternative to DE.

This implies a primordial angular frequency of: $\omega_{o}=2 \times 10^{-18} \mathrm{~Hz}$ and a corresponding time period of:

$T=3 \times 10^{18} s \sim 10 T_{H}$

where $T_{H}=10^{10}$ years is the Hubble time.

The angular momentum given by $J=M \omega R^{2}$ (this holds not only for disc but also for a spherical or hyspherical 3- 
spaceof the four dimensional space-time), is conserved. And this cosmic rotation can possibly impart rotation to galaxies, clusters, stellar systems, etc. as we shall see below.

Recent work on the study of thousands of spiral galaxies imaged by Sloan Digital Sky Survey does in fact indicate that the universe could be spinning [9].

For a galaxy, the angular momentum is given by:

$J_{g a l}=M_{g a l} \omega_{o} R_{g a l}^{2}$

where, $M_{\text {gal }}=10^{45} \mathrm{~g} ; R_{\text {gal }}^{2}=10^{47} \mathrm{~cm}^{2}$ and we assume the same primordial value of angular frequency; $\omega_{o}=2 \times 10^{-18} \mathrm{~Hz}$

This implies a galactic angular momentum of:

$J_{\text {gal }} \approx 10^{74}$ ergs.s $\sim 10^{100} \hbar$

which is the observed angular momentum of galaxies!

Similarly for clusters of galaxies, the angular momentum is given by:

$J_{\text {Clust }}=M_{\text {Clust }} \omega_{o} R_{\text {Clust }}^{2} \approx 10^{110} \hbar$

This is the observed angular momentum of large galaxy cluster.

(on a Hubble scale we have a corresponding $J \approx 10^{120} \hbar$ ) by:

In the case of the sun, the angular momentum is given

$J_{\text {Sun }}=M_{\text {Sun }} \omega_{o} R_{\text {Sun }}^{2} \approx 10^{50}$ ergs.s

which is only about $1 \%$ of the angular momentum of the solar system.

Considering the solar system we have:

$J_{\text {SolSys }}=M_{\text {SolSys }} \omega_{o} R_{\text {SolSys }}^{2} \approx 10^{52}$ ergs.S

which again matches observation.

The idea is that the initial solar nebula which condensed into the sun and planetary system also had its total angular momentum derived from the primordial rotation involving the same value of $\omega_{o}$.

Therefore we see that invoking primordial cosmic rotation can give rise to the observed rotation angular momenta of galaxies, galaxy clusters, stellar planetary systems, etc., the origin of which is otherwise not clearly understood $[3,5]$. It is remarkable that the same (universal) primordial value of $\omega_{o}$ was used in all the above cases.

\section{NON-IRROTATIONAL HYDRODYNAMIC FLOW AND VORTICES}

Here we have cosmological model involving the primordial rotation of the universe, invoked to understand the origin of the rotation or spin of objects over a wide range of masses from stars to galaxies. However this does not address the question of the distribution of the primordial angular momentum over the very large number of these objects. For instance, there are about a hundred billion stars in a typical large galaxy and a hundred billion galaxies in the universe.
It is clear that hydrodynamics and turbulence have to be invoked. Some models of galaxy formation have explicitly included those effects [10, 11]. Again we have models, where cosmic strings have played a role as 'seeds' to trigger galaxy formation $[12,13]$. The study of cosmic strings from the point of view of defects in condensed matter physics, has led to experiments in analogy with superfluids, wherein rotating a container of superfluid leads to the angular momentum being distributed in an array of vortices with quantized circulation [14]. For early work on relevance of superfluid vacuum state to phase transitions in the early universe see ref. [15].

In what follows, we shall invoke this property of nonirrotational hydrodynamic flow, to understand how the primordial rotation of the universe (as suggested in ref. [2] and earlier works) broken up into vortex line structures, can indeed lead to formation of a large number of galactic structures, which in turn can lead to equally large number of stellar objects (within a galaxy).

For a fluid with non-irrotational flow,

$\nabla \times \mathrm{v}_{\mathrm{S}} \neq 0$

In the case of a superfluid, equation (9) holds at singular line core of a quantised vortex line, and the quantum of circulation is governed by the Onsager-Feynman relation:

$\oint \mathrm{v} d r=n \frac{\hbar}{m_{S}}$

$\oint \mathrm{v} d r=\iint \nabla \times \mathrm{v}_{\mathrm{S}} d A$

Or for an angular velocity $\omega$ :

$\oint \omega r d r=n \frac{\hbar}{m_{S}}$

( $m_{S}$ is the mass of the particle)

For a rotating container, we have: $2 \pi r . v=n \hbar / m_{S}$, giving the number of vortices per unit area as $2 \omega m_{S} / \hbar$, and the total number of vortices as:

$N=\frac{\omega \cdot 2 \pi r^{2} m}{\hbar}$

In actual experiments, for a container rotating at 1000rps, about $2 \times 10^{6}$ vortices $/ \mathrm{cm}^{2}$ were formed in agreement with the above formulae.

Following the numbers given in ref. [2], we can estimate the number of galaxy mass 'vortices' in a region (container) of the size of the universe $\left(R=10^{28} \mathrm{~cm}\right)$ with a primordial angular velocity $\omega=2 \times 10^{-18} \mathrm{rad} / \mathrm{s}$. $\hbar$ would be replaced by the typical galaxy angular momentum of $\sim 10^{74}$ ergs.s.

This gives the number of galaxies which would have been formed as:

$N_{g a l}=\frac{2 \times 10^{-18} \times 10^{57} \times 10^{45}}{10^{74}} \approx 10^{11}$ 
Here we have used for a typical vortex mass, a galaxy mass, $m \sim 10^{45} \mathrm{~g}$, and $R^{2} \sim\left(3 \times 10^{28} \mathrm{~cm}\right)^{2}=10^{57} \mathrm{~cm}^{2}$

This is the actual number of galaxies in the universe. For an individual galaxy, the number of substructures (stars in this case), is again given using equation (12) but now, with $m \sim 10^{33} \mathrm{~g}, \quad R \approx 10^{23} \mathrm{~cm}$ (typical galaxy size region), $J \approx 10^{52}$ erg.s (typical for a star):

$N_{\text {stars }}=\frac{2 \times 10^{-18} \times 10^{46} \times 10^{33}}{10^{52}} \approx 10^{11}$

Thus typically $\sim 10^{11}$ stars form in individual galaxies. So apart from explaining the observed angular momenta of galaxies and stars, from a universe in primordial rotation, we are also able to obtain the numbers of galaxies and stars by using the hydrodynamic vortex analogy for which there is strong experimental laboratory evidence (i.e. for equations (11) and (12)).

Including the $\Lambda$ term equation (1) becomes:

$\frac{\dot{R}^{2}}{R^{2}}-\frac{8 \pi G \rho}{3}+\omega^{2}+\frac{\Lambda c^{2}}{3}=0$

The rotation can produce an acceleration analogous to that of DE. This acceleration is given by:

$\omega^{2} R_{U n i v} \approx 10^{-7} \mathrm{cms}^{-2}$

which is exactly the expected value of acceleration required to explain the accelerated expansion:

$\ddot{R}=\frac{4 \pi G \rho R}{3} \sim 10^{-7} \mathrm{cms}^{-2}$ have:

For the DE term to be comparable to the rotation we

$\frac{\Lambda c^{2}}{3}=\omega^{2} \Rightarrow \Lambda=\frac{3 \omega^{2}}{c^{2}} \sim 10^{-56} \mathrm{~cm}^{-2}$

which is precisely the present observed value. Thus a cosmological model with a large scale primordial rotation term of this order can give an accelerating universe mimicking a dark energy term.

Even on a cosmic scale of $\approx 10^{25} \mathrm{~cm}$, the deviation from isotropy will be of the order of $\sim\left(\frac{10^{25}}{10^{28}}\right)^{2} \sim 10^{-6}$, which is one order less than the sensitivities of the current probes $10^{-5}$. Even such a primordial rotation if it exists would be barely detectable.

Since the angular momentum $J$, is conserved, rotation will be dominant during the radiation era, since both $\omega^{2}$ and $\rho_{\text {rad }}$ will fall off as $1 / R^{4}$, where as in matter dominated era, $\omega^{2}$ falls off at a faster rate.

For a whole hierarchy of objects to be gravitationally bound, their gravitational (binding) self energy density should be at least equal or exceed the background cosmological gravitational self energy density (which again equals the critical matter density) [16-20]. This implies that, from the equation:

$\frac{G M^{2}}{R^{4}} \approx \frac{\Lambda c^{4}}{8 \pi G}$

$\frac{M}{R^{2}}=\frac{c^{2}}{G} \sqrt{\Lambda}$

That is:

$R^{2}=\frac{G M}{c^{2}} \frac{1}{\sqrt{\Lambda}}$

The angular momentum is then given by:

$J=M \omega R^{2}=M \omega_{o} \frac{G M}{c^{2}} \frac{1}{\sqrt{\Lambda}}$

Thus [21]:

$J \propto M^{2}$

This relation, as pointed out earlier in several papers [22, $23]$ holds for a very wide range of celestial objects. This relation has often been difficult to understand although it is empirically well established [24].

Here apart from getting reasonable values for the angular momenta of a wide range of objects we have a natural explanation for this quadratic scaling with mass. The value of the constant in the proportionality relation is related to $\omega_{o}$ above and works out to the right order of magnitude $\left(\sim 10^{-17}\right)$.

\section{TORSION AND EFFECTIVE COSMOLOGICAL CONSTANT}

Also the spin densities ( $\sigma=$ spin/volume) for a range of objects work out to be the same. For an electron, the classical radius is $\sim 3 \times 10^{-13} \mathrm{~cm}$, and the spin density is given by:

$\sigma_{e}=\frac{0.5 \hbar}{\left(\frac{4}{3} \pi r_{e}^{3}\right)} \sim 10^{9}$ ergs.s $/ c c$

Similarly for a proton:

$\sigma_{P} \sim 10^{9}$ ergs.s $/ \mathrm{cc}$

Also for the solar system we have:

$\sigma_{\text {Sollys }} \sim 10^{9}$ ergs.s $/ c c$

For a galaxy, the angular momentum is (from equation (5)) $J_{g a l} \sim 10^{100} \hbar$, the spin density is then given as:

$\sigma_{g a l}=\frac{10^{100} \hbar}{\left(\frac{4}{3} \pi R_{g a l}^{3}\right)} \sim 10^{9}$ ergs.s $/ c c$

And for the universe, $J_{U n i v} \sim 10^{120} \hbar$, and the corresponding spin density is then given as:

$\sigma_{\text {Univ }}=\frac{10^{120} \hbar}{\left(\frac{4}{3} \pi R_{H}^{3}\right)} \sim 10^{9}$ ergs.s $/ c c$ 
From equations (24) - (28), we see that within an order of magnitude spin density i.e. spin or angular momentum per unit volume is same for all structures, from elementary particles to the universe. The spin density in Einstein-Cartan theory is related to torsion $(Q)$ of background space-time as:

$Q \propto \sqrt{\text { curvature }}$

The torsion concept to justify repulsive gravity has also been considered by many authors $[25,26]$

The torsion is given by:

$Q=\frac{4 \pi G \sigma}{c^{3}}$

where the spin density:

$\sigma=\frac{J}{R^{3}}$

and $R$ is the scale size of background space.

From equations (24) - (28), we have:

$\sigma=10^{9}$ ergs.s $/ c c$

Therefore the torsion is given by:

$Q=\frac{4 \pi G \sigma}{c^{3}} \sim 10^{-28} \mathrm{~cm}^{-1}$

And the background curvature

$\sim Q^{2} \approx 10^{-56} \mathrm{~cm}^{-2}$

Torsion gives a modification of Poisson equation as: [27]

$\nabla^{2} \phi=4 \pi G\left(\rho-G \sigma^{2}\right)$

and acts opposite to gravity and is a repulsive term. Indeed, the $G \sigma^{2}$ term (for a constant $\sigma$ as implied by equations $(24)-(28))$ gives rise to a potential $\sim G \sigma^{2} r^{2}$, growing with $r$ like a cosmological constant term. This is precisely the order of the observed $\Lambda$.

Hence it could be a possible candidate for dark energy!

At present epoch, $\frac{G \sigma^{2}}{c^{4}}\left(\sim 10^{-29} \mathrm{~g} / c c\right)>\rho$,

where

$\rho \sim 3 \times 10^{-30} \mathrm{~g} / \mathrm{cc}$.

The spin density in Cartan's equation plays the role of a repulsive potential. The non-minimal spin-spin interaction term arising out of the interaction of the interaction of a Dirac spin or particle with the vierbein gravitational field gives rise to an "effective cosmological constant term", as indicated in refs [28-30].

Again it has been shown that the so called teleparallel gravity (first introduced by Einstein as a possible alternative to GR) which uses the torsion instead of the curvature in the gravitational action is equivalent to a theory with a cosmological constant [27].

Recent studies [31] on the fluctuations in the cosmic microwave background have revealed a coherent bulk flow of clusters of galaxies on a large cosmic scale. This flow (termed the dark flow) is difficult to explain with the current cosmological models. But invoking a primordial rotation with the same value of $\omega_{o}$ can account for the observed peculiar velocities (which on the scales of $\sim 300 M p c$ could give rise to coherent bulk flows of $\sim 3 \times 10^{4} \mathrm{Km} / \mathrm{s}$, suggesting increase of large scale velocities with distance) of these clusters of galaxies.

So the background torsion due to a universal spin density can not only give rise to angular momenta for all structures but also provide a background 'centrifugal term' acting as a repulsive gravity accelerating the universe, mimicking the effective cosmological constant or accelerating dark energy of the observed magnitude.

\section{ACKNOWLEDGEMENT}

None declared.

\section{CONFLICT OF INTEREST}

None declared.

\section{REFERENCE}

[1] Trimble V. The angular momentum-vs-mass relation for spectroscopic binaries. Astrophys Space Sci 1984; 104: 133.

[2] Sivaram C, Arun K. Some aspects of rotational and magnetic energies for a hierarchy of celestial objects. Astrophys Space Sci 2012 337: 767-771.

[3] Gamow G. Rotating universe. Nature 1946; 158: 549.

[4] Gödel K. An example of a new type of cosmological solutions of einstein's field equations of gravitation. Rev Mod Phys 1949; 21 : 447-50.

[5] Barrow JD, Juszkiewicz R, Sonoda DH. Universal rotation - How large can it be? Mon Not R Astron Soc 1985; 213: 917.

[6] de Sabbata V, Sivaram C. Particle magnetic moments in KaluzaKlein strong gravity. Nuovo Cimento B 1990; 105: 1181.

[7] de Sabbata V, Sivaram C. Torsion and inflation. Astrophys Space Sci 1991; 176: 141.

[8] de Sabbata V, Sivaram C. Torsion, wormholes, and the problem of the cosmological constant. Int J Theor Phys 1991; 30:123.

[9] Longo M. Detection of a Dipole in the Handedness of Spiral Galaxies with Redshifts z 0.04. Phys Lett B 2011; 699: 224.

[10] Krishan V, Sivaram C. Clustering of galaxies by inverse cascade in a turbulent medium. Mon Not R Astron Soc 1991; 250: 157.

[11] Bournaud F. In: J. Alves, Ed, Computational Star Formation: Proceedings IAU Symposium. Cambridge: Cambridge University Press 2010; pp.491-498.

[12] Sivaram C. Proceedings of the 20th International Cosmic Ray Conference Moscow. 1987; Vol. 5, p.167.

[13] Manías MV, Naón CM, Schaposnik FA, et al. Non-abelian charged vortices as cosmic strings. Phys Lett B 1986; 171: 199.

[14] Finne AP, Eltsov VB, Hänninen R, et al. Dynamics of vortices and interfaces in superfluid 3He. Rep Prog Phys 2006; 69: 3157.

[15] Volovik GE. Theory of quantized vortices in rotating superfluid ${ }^{3}$ He. Physica B 1984; 126: 34.

[16] Sivaram C. Scale transformations and evolution of the early universe. Astrophys Space Sci 1993; 207: 317.

[17] Sivaram C. Mond, dark matter and the cosmological constant. Astrophys Space Sci 1994; 219: 135.

[18] Sivaram C. Some further effects of discrete scale transformations. Astrophys Space Sci 2000; 271: 321.

[19] Sivaram C. In: Saha SK, Rastogi VK, Eds. The cosmological constant as a unifying link: $21^{\text {st }}$ Century Astrophysics. New Delhi: Anita Publications 2005; p.16.

[20] Sivaram C. Scaling relations for self-similar structures and the cosmological constant [astro-ph/0801.1218]. Available from: http://arxiv.org/abs/0801.1218, [8 January 2008].

[21] Wesson PS. Clarification of the angular momentum $/ \mathrm{mass}$ relation $\left(\mathrm{J}=p \mathrm{M}^{2}\right)$ for astronomical objects. Astron Astrophys 1983; 119: 313 . 
[22] Sivaram C. Cosmic scrings and cobjects. Nature 1987; 327: 108.

[23] de Sabbata V, Sivaram C. Magnetic fields in the early universe. Nuovo Cimento 1988; 102: 107.

[24] Sivaram C. In: de Sabbata V, Karade TM, Eds. Relativistic Astrophysics and Cosmology. Singapore: World Scientific 1984; p. 228.

[25] de Sabbata V, Sivaram C. The need for an energy-dependent torsion-coupling constant in the early universe. Astrophys Space Sci 1989; $158: 347$

[26] de Sabbata V, Sivaram C. Torsion, string tension, and topological origin of charge and mass. Found Phys Lett 1995; 8: 375.

[27] de Sabbata V, Sivaram C. Spin and torsion in gravity. Singapore: World Scientific 1994.
[28] Isham CJ, Salam A, Strathde J. Influence of $\mathrm{f}$ gravity on gravitational collapse. Nature 1973; 244: 82.

[29] Sivaram C, Sinha KP, Lord EA. $f$ Gravity and gravitational singularities. Nature 1974; 249: 640.

[30] Sivaram C, Sinha KP, Lord EA. Role of f-gravity in cosmological models. Curr Sci 1975; 44: 143.

[31] Kashlinsky A, Atrio-Barandela F, Kocevski D, et al. A measurement of large-scale peculiar velocities of clusters of galaxies: results and cosmological implications. Astrophys J 2008; 686: 49 .

(c) Sivaram and Arun; Licensee Bentham Open.

This is an open access article licensed under the terms of the Creative Commons Attribution Non-Commercial License (http://creativecommons.org/licenses/by-nc/3.0/) which permits unrestricted, non-commercial use, distribution and reproduction in any medium, provided the work is properly cited. 